ORIGINAL ARTICLE

\title{
Crisis management during anaesthesia: bradycardia
}

\author{
L M Watterson, R W Morris, R N Westhorpe, J A Williamson
}

Qual Saf Health Care 2005;14:e9 (http://www.qshc.com/cgi/content/full/14/3/e9). doi: 10.1136/qshc.2002.004481

See end of article for authors' affiliations

......................

Correspondence to: Professor W B Runciman, President, Australian Patient Safety Foundation, GPO Box 400, Adelaide, South Australia 5001 Australia; research@apsf. net.au

Accepted 11 January 2005
Background: Bradycardia in association with anaesthesia may have many potential causes and associated conditions, some rare and/or obscure. A prompt appropriate response is important as some homeostatic mechanisms may be impaired under anaesthesia.

Objectives: To examine the role of a previously described core algorithm "COVER ABCD-A SWIFT $\mathrm{CHECK}^{\prime \prime}$, supplemented by a specific sub-algorithm for bradycardia, in the management of bradycardia occurring in association with anaesthesia.

Methods: The potential performance of this structured approach for each of the relevant incidents among the first 4000 reported to the Australian Incident Monitoring Study (AIMS) was compared with the actual management as reported by the anaesthetists involved.

Results: From the first 4000 incidents reported to AIMS, 265 reports which described bradycardia during anaesthesia were extracted and studied. Bradycardia was associated with hypotension in $51 \%$ of cases, cardiac arrest in $25 \%$ of cases and hypertension in one case. In $22 \%$ of reports apparent desaturation or an abnormality of ventilation was described. Bradycardia was caused by drug events (28\%), airway related events (16\%), autonomic reflexes (14\%), and regional anaesthesia (9\%). Airway and drug events caused $75 \%$ of cases involving children. It was considered that, correctly applied, the core algorithm COVER would have diagnosed 53 cases $(20 \%)$ and led to corrective management in $45(85 \%)$ of these; this included an important subset of airway and ventilation problems. Completion of COVER ABCD-A SWIFT CHECK followed by the specific sub-algorithm for bradycardia would have resulted in diagnosis and appropriate management in all but two cases. It would have led to earlier recognition of the problem and/or better management in 11 cases $(4 \%)$ when compared with the actual management described in the reports.

Conclusion: Steps should be taken to manage bradycardia whilst associated conditions are managed concurrently. Analysis of cardiac rhythm should not be pursued to the exclusion of supportive therapy. The use of a structured approach in the management of bradycardia associated with anaesthesia is likely to improve management in the small percentage of cases in which the diagnosis of the cause may be missed or delayed.
C ardiac output is determined by the product of heart rate and left ventricular stroke volume. The contribution of both atrial and ventricular contraction to the stroke volume underlies the significance of normal cardiac rhythm and conduction in the generation of cardiac output. Bradycardia may result in an insufficient rate to sustain cardiac output and hence oxygen delivery to tissue beds. This is particularly so in the case of young children in whom cardiac output is more affected by changes in heart rate than stroke volume. In older patients bradycardia may reflect an ischaemic myocardium which is unable to maintain a stable electrical rhythm as a result of systemic hypotension or hypoxaemia. A prompt appropriate response to bradycardia under anaesthesia is important as some causes are rare and/ or obscure and homeostatic mechanisms may be impaired by anaesthetic agents. It was decided to examine the role of a structured response to bradycardia under anaesthesia as there are many potential causes and associated conditions.

In 1993 a "core" crisis management algorithm represented by the mnemonic COVER ABCD-A SWIFT CHECK (the AB precedes COVER for the non-intubated patient) was proposed as the basis for a systematic approach to any crisis during anaesthesia where it is not immediately obvious what should be done or where actions taken have failed to remedy the situation. ${ }^{1}$ This was validated against the first 2000 incidents reported to the original Australian Incident Monitoring Study (AIMS). AIMS is an ongoing study which involves the voluntary anonymous reporting of any unintended incident which reduced or could have reduced the safety margin for a patient. ${ }^{2}$

It was concluded that, if this algorithm had been correctly applied, a functional diagnosis would have been reached within 40-60 seconds in 99\% of applicable incidents, and that the learned sequence of actions recommended by the COVER portion would have led to appropriate steps being taken to handle $60 \%$ of problems relevant to this portion of the algorithm. ${ }^{1}$ This study also showed that the $40 \%$ of problems represented by the remainder of the algorithm ABCD-A SWIFT CHECK were not always promptly diagnosed or appropriately managed..$^{1-3}$ It was decided that it would be useful for these remaining problems to develop a set of subalgorithms in an easy-to-use crisis management manual. ${ }^{4}$ This study reports on the potential place of the COVER ABCD-A SWIFT CHECK algorithm in the diagnosis and initial management of bradycardia, provides an outline of a specific crisis management sub-algorithm for bradycardia during anaesthesia, and provides an indication of the potential value of using this structured approach.

\section{METHODS}

Of the first 4000 incidents reported to AIMS, those which made reference to bradycardia or bradydysrhythmia were extracted and analysed with respect to the following criteria: anaesthetic technique, patient's ASA grading, phase of anaesthesia at the time of presentation, presenting features (cardiac rhythm, blood pressure, oxygen saturation), 


\section{BRADYCARDIA}

\section{EMERGENCY MANAGEMENT}

Complete COVER ABCD-A SWIFT CHECK

Do not hesitate to treat as Cardiac Arrest $\rightarrow$ page $38^{*}(1)^{* *}$

Ensure adequacy of oxygenation and ventilation (2)

If hypotensive (3)

Inform and interrogate the surgeon

Turn off vaporiser

Crystalloid $10 \mathrm{ml} / \mathrm{kg}$ bolus and repeat if necessary

Atropine $0.01 \mathrm{mg} / \mathrm{kg}$ (that is $0.6 \mathrm{mg}$ for an adult)

Consider adrenaline $0.001 \mathrm{mg} / \mathrm{kg}$ IV bolus $(1 \mathrm{ml}$ of $1: 10,000)$

Followed if necessary by an infusion of adrenaline starting at

$0.00015 \mathrm{mg} / \mathrm{kg} / \mathrm{min}(1 \mathrm{ml} / \mathrm{min}$ of $1 \mathrm{mg}$ in $100 \mathrm{ml})$

Increase monitoring - ECG, arterial line, CVP

Consider external pacemaker (transvenous or transcutaneous)

REVIEW AND TREAT PROBABLE CAUSES: (4)

\section{Drugs (5)}

Inhalational agent overdose. Consider also suxamethonium,

induction agents, neostigmine, and opioids. Check drugs given by

surgeon.

Airway (2)

Hypoventilation $\rightarrow$ page $20^{*}$

Hypoxia $\rightarrow$ page $26^{*}$

Vagal Reflexes (6)

Cease stimulation

Regional Anaesthetic (7)

Consider: Vasodilation, bradycardia, respiratory failure.

Ensure: Volume loading, vasopressors (early adrenaline), airway

support, left lateral displacement during pregnancy.

Surgical Factors (8)

Consider: IVC compression, pneumoperitonium, retractors position.

Ensure: Surgeon aware.

Undetected Blood Loss (9)

Improve IV access, fluid replacement, cross match.

Cardiac Event (4)

Consider: Tension pneumothorax, haemothorax, tamponade,

embolism (gas, amniotic or thrombus), sepsis, myocardial depression

(from drugs, ischaemia, electrolytes, trauma).

Ensure: Review of appropriate sub-algorithms.

The sub-algorithm forms a facing page of the

Crisis Management Manual 13.

* Page references refer to the Crisis Management Manual ${ }^{13}$.

** Numbers in brackets refer to Notes in the right hand panel.

Figure 1 Bradycardia.

presumed precipitating cause, management and outcome. The algorithm COVER-A SWIFT CHECK algorithm, described elsewhere in this series of articles, ${ }^{4}$ was applied to each relevant report to determine the stages at which the problem might have been diagnosed and to confirm that activating the COVER portion would have led to appropriate initial steps being taken.

As bradycardia is not adequately dealt with by this algorithm, a specific sub-algorithm for bradycardia was developed (see fig 1) and its putative effectiveness was tested against the reports. How this was done is described elsewhere in this series of articles. ${ }^{4}$ The reports were used in conjunction with contemporary guidelines on resuscitation ${ }^{5-9}$ to generate a differential diagnosis and management algorithm which was especially relevant to anaesthesia. The potential value of this structured approach-that is, the application of COVER ABCD-A SWIFT CHECK to the diagnosis and management of the problem followed by the application of the bradycardia sub-algorithm-was assessed

\section{FURTHER CARE}

If the situation warrants:

Continue the adrenaline infusion, titrate against heart rate and blood pressure.

Consider transvenous or transcutaneous pacing

Consider an urgent cardiology consult

NOTES:

It was judged that correct use of the algorithm would have led to earlier recognition of the problem and/or better management in $4 \%$ of 295 relevant incidents reported to AIMS.

(1) $25 \%$ presented as cardiac arrest.

(2) Airway problems were a factor in $19 \%$ of cases.

(3) $51 \%$ of cases were associated with hypotension.

(4) See left hand panel for the most common causes. Other important causes include myocardial events (particularly inferior ischaemia/infarction), anaphylaxis, pneumothorax and air/other embolism. At least one of these factors was responsible in $5 \%$ of cases. Multiple causes occurred in $22 \%$ of cases.

(5) Drugs were a factor in $40 \%$ of cases. Those most commonly implicated were inhalational agents $(26 \%)$, opioids (16\%), intravenous anaesthetics (14\%) and suxamethonium (9\%). Other drugs included muscle relaxants, anticholinesterases, metaraminol and phenytoin.

(6) Vagal reflexes were a factor in $14 \%$ of cases, usually following a surgical stimulus. The stimulus should be ceased and the problem usually resolves.

(7) Regional anaesthesia was a factor in $9 \%$ of cases.

(8) Surgical factors were present in $4 \%$ of cases.

(9) In addition to intraoperative losses, preoperative blood loss and occult losses (i.e. into the chest cavity) were also reported. Hypovolaemia was a factor in $3 \%$ of cases.

These notes comprise a reverse side of a page of the Crisis Management Manual ${ }^{13}$. in the light of the AIMS reports by comparing its potential effectiveness for each incident with that of the actual management as recorded in each report. In addition to determining a difference between the two approaches with respect to likelihood of correct diagnosis and management, it was of particular interest during this part of the project to evaluate if delays in diagnosis and overall outcome could be improved with the application of the sub-algorithm.

\section{RESULTS}

Of the first 4000 incidents reported to AIMS, there were 265 reports which contained the words "bradycardia" or "bradydysrhythmia"; what constituted an incident was a decision made by the original reporter. These incidents occurred across a wide range of surgical groups. The patients' ASA grades were available in 254 reports (table 1).

Bradycardia occurred during all phases of anaesthesia, but most commonly during the maintenance phase (table 2 ). 
Table 1 ASA grades $(n=265)$

\begin{tabular}{lcl}
\hline ASA grade & No $(\%)$ adults & No $(\%)$ children* \\
\hline II & $69(26)$ & $22(8)$ \\
III & $64(24)$ & $11(4)$ \\
II & $58(22)$ & $6(2)$ \\
V & $15(6)$ & $2(<1)$ \\
Not specified & $5(2)$ & $2(<1)$ \\
Total & $11(4)$ & - \\
\hline *Children aged $<14$ years. & $222(84)$ & $43(16)$ \\
\hline
\end{tabular}

\section{Aetiology}

The attributed causes of bradycardia are shown in table 3. Overall, a total of 319 causative events were described for the 265 incidents involving bradycardia. The most common causes of bradycardia were drug events $(28 \%)$, problems with the airway or ventilation (16\%), autonomic reflexes (14\%), and epidural or spinal anaesthesia (9\%). A putative cause was not determined in 26 cases $(8 \%)$. In addition, all cases of bradycardia are compared with subgroups based on associated blood pressure and are also cross referenced with datasets from the AIMS data relating to all cases of hypotension (table 3). The aim of this was to identify patterns which may indicate treatment when bradycardia coincided with abnormalities of blood pressure. Airway related events appear more likely to be associated with normotension, and neuraxial blockade appears more likely to be associated with hypotension. It also appears that cardiopulmonary events causing bradycardia are more likely than other causes to be associated with cardiac arrest.

Drug events were reported as a likely cause in 89 reports. In many cases it was not possible to clearly distinguish the specific contribution of any particular drug when drugs were given in combination. In this event, each drug was described as a drug event. Overall, 129 drug events were nominated. A breakdown of drug related bradycardia is shown in table 4 .

The most commonly cited drugs were inhalational agents $(n=34)$, opiates $(n=20)$, intravenous induction agents $(\mathrm{n}=18)$, and suxamethonium $(\mathrm{n}=12)$. Enflurane and thiopentone were the most commonly described inhalational and intravenous agents, respectively. The time of collection of reports predated the wider usage of newer agents. Fentanyl was the most commonly described opiate. Its use was described in combination with other agents on all but two of a total of 11 occasions. Suxamethonium caused bradycardia after a repeat dose in $50 \%$ of cases. Drug errors and vaporiser incidents accounted, respectively, for nine and two of the total drug events described. A drug error was defined as the inadvertent administration of an agent and included syringe and ampoule swaps and residual agents in intravenous lines. Vaporiser incidents were identified whenever the reporter was unaware that an agent was being administered at all or in the concentration detected. This included incorrect settings and administration of multiple agents due to the absence of interlock devices.

Problems with the airway or ventilation were described in 58 reports. These included desaturation on pulse oximetry, difficulty with ventilation, clinical cyanosis, or symptoms of dyspnoea. On eight occasions no specific cause of the problem could be found with the airway; it is possible that the desaturation represented an artefact or true desaturation resulting from altered ventilation-pulmonary blood flow matching. The remaining 50 cases were associated with real airway abnormalities. This represented 19\% of all reports describing bradycardia and $16 \%$ of total causes described when multiple causes were accounted for. The specific nature of these airway events is shown in table 5 .

\begin{tabular}{lc}
\hline Table 2 & Bradycardia: \\
\hline Phase & $\%$ \\
\hline Pre-induction & 5 \\
Induction & 15 \\
Maintenance & 61 \\
Emergence & 8 \\
Postoperative & $<1$ \\
Recovery & 8 \\
Not specified & 2 \\
Total & 100 \\
\hline
\end{tabular}

Importantly, no factors emerged which would enable the reporter to differentiate those caused by artefact (such as monitor malfunction) from real airway events without a formal assessment of the airway.

Vagal reflexes were considered to have contributed to bradycardia in 45 reports, which represented $14 \%$ of the 319 causes described. These reflexes were surgically induced in 36 cases. The majority of these were attributed to either vagal stimulation in the abdominal cavity or pelvis $(n=13)$ or the oculocardiac reflex $(n=12)$. Anaesthetic causes included laryngoscopy $(\mathrm{n}=4)$ and intravenous cannulation $(\mathrm{n}=2)$.

Surgical events were associated with 13 reports $(5 \%)$. These included reaming, cementing or manipulating fractured hips $(\mathrm{n}=4)$, manipulation of wires or catheters in central veins $(\mathrm{n}=4)$, and pneumoperitoneum $(\mathrm{n}=2)$.

\section{Diagnosis}

Bradycardia was described with abnormalities of blood pressure in 202 reports (76\%). Bradycardia in association with a state of cardiac arrest, as defined by the absence of a peripheral pulse or the presence of a cardiac rhythm incompatible with a cardiac output (sinus arrest, asytole), was described in 67 reports $(25 \%)$. Within this group, 24 cases involved degeneration from an initial state of hypotension into a pulseless state. Bradycardia was associated with hypotension in 134 cases $(51 \%)$ and hypertension in one $(<1 \%)$. There was no significant change in blood pressure in the remaining 63 cases $(24 \%)$.

The prevailing rhythm when bradycardia was detected was specifically described as sinus rhythm in 74 reports. In another 135 reports sinus bradycardia was inferred from the narrative (table 6). Together, these account for $79 \%$ of cases.

Bradycardia degenerated into asystole in 19 cases. However, on a number of occasions the narrative suggested that sinus arrest was the actual rhythm. The reported rhythm was recorded on these occasions. The remaining 56 cases $(21 \%)$ specified a non-sinus rhythm.

\section{Management}

The management of bradycardia as described in the narrative of the reports was classified as either conventional or nonconventional based upon the guidelines of the American Heart Association (AHA), ${ }^{5}$ the International Committee on Resuscitation, ${ }^{6}$ and the Australian Resuscitation Council (ARC). ${ }^{7-9}$ It was subdivided into five grades which ranged from simple supportive measures to tertiary management (table 7)

Management was specified in 225 cases; in 25 of these it was classified as non-conventional. The most common reasons for this related to resuscitation with agents no longer recommended by AHA or ARC. Examples included the use of calcium $(n=5)$ and isoprenaline $(n=7)$, the omission of adrenaline when indicated $(n=9)$, an excessive dose or inappropriate route of administration of atropine $(\mathrm{n}=2)$, and DC cardioversion for asytole $(\mathrm{n}=1)$. Vasopressors were used in nine cases of cardiac arrest and 36 cases of hypotension. 
Table 3 Causes of bradycardia expressed as percentage of total causes $(\mathrm{N}=319)$

\begin{tabular}{|c|c|c|c|c|c|}
\hline Causes & Hypotension & Bradycardia & H-brady & CA-brady & $\mathrm{N}$-brady \\
\hline Allergy & 7 & 2 & 3 & 2 & 0 \\
\hline Airway problem & 4 & 16 & 7 & 10 & 31 \\
\hline Hypovolaemia & 9 & 3 & 4 & 5 & 0 \\
\hline Regional anaesthesia & 17 & 9 & 16 & 9 & 7 \\
\hline Drug effects & 26 & 28 & 30 & 31 & 19 \\
\hline Cardiopulmonary & 5 & 5 & 4 & 10 & 3 \\
\hline Reflexes & 6 & 14 & 14 & 13 & 15 \\
\hline Posture & 1 & 1 & 1 & 1 & 0 \\
\hline Sepsis & 1 & 2 & 1 & 5 & 0 \\
\hline Surgery & 2 & 4 & 2 & 7 & 6 \\
\hline Pre-existing condition & 1 & 3 & 3 & 2 & 2 \\
\hline Central nervous system & 0 & 1 & 0 & 2 & 0 \\
\hline Endocrine & 1 & 3 & 2 & 0 & 2 \\
\hline Monitoring error & 2 & 1 & 1 & 0 & 2 \\
\hline Other & 1 & 0 & 0 & 0 & 6 \\
\hline Not determined & 16 & 8 & 11 & 3 & 7 \\
\hline Total (\%) & 99 & 100 & 99 & 100 & 100 \\
\hline
\end{tabular}

$\mathrm{H}$-brady, bradycardia in association with hypotension; CA-brady, bradycardia in association with cardiac arrest; N-brady, bradycardia without associated hypotension.

The most commonly used vasopressors were ephedrine $(n=17)$, metaraminol $(n=15)$, or a combination of the two $(n=4)$. External cardiac compression (ECC) was specified as given in only 45 of the 67 reports describing cardiovascular collapse. It was assumed to have been given whenever the report specified either the use of "resuscitation" or "cardiac life support". The remaining cases were non-conventional by definition on the basis of $\mathrm{AHA}^{5}$ and $\mathrm{ARC}^{79}$ resuscitation guidelines for management of pulseless patients. It is acknowledged that, on some occasions, ECC may have been instituted but not reported.

\section{Outcome}

The outcome from bradycardia is shown in table $8 ; 37 \%$ of reports described a poor outcome. Reports were considered to have a poor outcome if they were not resolved by the completion of the normal recovery period. An exception to this was ECG evidence of myocardial ischaemia or other cardiac events which may have resolved but often resulted in overnight monitoring.

\section{Table 4 Drug related causes of bradycardia}

\begin{tabular}{lc}
\hline Drugs & No \\
\hline Inhalational agents* & 34 \\
Opioids $†$ & 20 \\
IV anaesthetic agents $\ddagger$ & 18 \\
Suxamethonium & 12 \\
Muscle relaxants & 7 \\
Anticholinesterase & 6 \\
Metaraminol & 5 \\
Local anaesthetic§ & 3 \\
Benzodiazepines & 3 \\
Vaporiser incident & 2 \\
Miscellaneous** & 10 \\
Drug errort† & 9 \\
Total & 129 \\
\hline
\end{tabular}

Note: several drugs may be given in combination. Examples of specific drug related causes of bradycardia: *enflurane (17), halothane (11), isoflurane (3), not specified (3); †fentanyl (11), pethidine as premedication (6), morphine (2), omnopon (1); thiopentone (6), propofol (4), not specified (8); $\top$ second dose (5); §overdose (1), toxicity (1); **droperidol (2), vancomycin (1), phenytoin (1), hydrallazine (1), methylprednisolone (1), digoxin (1), ornithine 8vasopressin POR8 (1), beta-blockers (1), promethazine (1); ††syringe swap: suxamethonium (1), fentanyl (1), (2), metaraminol (1), not specified (2), residual in IV line: suxamethonium (1), morphine (1).

\section{Paediatric cases}

Bradycardia occurring in children under the age of 14 years was described in 43 reports (16\% of all reports). The distribution of ASA grading was similar to that of adults. Airway and drug events were responsible in $47 \%$ and $28 \%$ of paediatric cases, respectively. The haemodynamic profile was different in children than in adults. In $51 \%$ of cases there was no abnormality of blood pressure. This was attributed to the high incidence of airway events in which event hypoxaemia was associated with rapid deterioration in heart rate. This pattern has been identified in earlier studies. ${ }^{8}$ A smaller percentage of cases was associated with hypotension $(21 \%)$. In contrast, cardiac arrest was described in $32 \%$. In this group, drug events and a pre-existing condition were the most common causes. Non-sinus rhythms were reported in $12 \%$ of children $(n=7)$ compared with $16 \%$ overall. A good outcome was reported in only $48 \%$ of cases compared with $63 \%$ overall. However, there was a higher percentage of outcomes not specified in children $(25 \%)$ than in the total group (12\%).

\section{Verification of the sub-algorithm}

When COVER ABCD-A SWIFT CHECK was applied to each report it was considered that the problem would have been detected in all cases either at the $\mathrm{C} 1$ stage of COVER or at $\mathrm{C}$ of ABCD. Fifty three cases $(20 \%)$ were diagnosed of which 45 $(85 \%)$ would also have received correct management as a result of applying the core algorithm COVER. The majority of

Table 5 Causes of airway/ventilation/ oxygenation related bradycardia

\begin{tabular}{lc}
\hline Causes & No of events \\
\hline Laryngospasm & 10 \\
Hypoventilation (drug induced and other) & 9 \\
$\mathrm{O}_{2}$ delivery/circuit/ETT & 5 \\
Airway obstruction & 4 \\
Aspiration & 3 \\
Failed or difficult intubation & 3 \\
Spinal/epidural & 3 \\
Hypoxaemia (cause not determined) & 3 \\
Oesophageal intubation & 2 \\
Embolism & 2 \\
Allergy & 2 \\
Bronchoconstriction & 1 \\
Pneumothorax & 1 \\
Total & 50 \\
\hline ETT, endotracheal tube. &
\end{tabular}




\begin{tabular}{|lr|}
\hline Table 6 Bradycardia: cardiac rhythm \\
\hline Rhythm & No \\
\hline Sinus & \\
Sinus bradycardia (specified) & 43 \\
?Sinus bradycardia & 135 \\
Sinus arrest & 12 \\
Sinus bradycardia (asytole) & 19 \\
Non-sinus & 29 \\
Nodal bradycardia & 1 \\
Nodal bradycardia (asytole) & 13 \\
Complete heart block & 4 \\
Idioventricular complexes & 3 \\
Atrial fibrillation & 1 \\
Atrial tachycardia with block & 3 \\
Bigeminy & 2 \\
Non-sinus (not determined) & 265 \\
Total & \\
\hline
\end{tabular}

these dealt with circuit problems Vl $(n=3)$, vaporiser incidents V2 $(\mathrm{n}=34)$, problems arising from monitors $\mathrm{Rl}$ $(\mathrm{n}=5)$, equipment malfunction or errors with non-anaesthetic drugs R2 $(\mathrm{n}=11)$. The remaining cases required completion of the sub-algorithm for bradycardia; this failed to provide a diagnosis or appropriate corrective management in two cases. Asymmetrical radial pulses and an underreading central venous pressure monitor were responsible in these situations. It was felt that the algorithm performed better than the reporter in 11 cases $(4 \%)$ : missed or delayed diagnoses $(n=7)$, inappropriate use of drugs $(n=3)$, and failure to improve monitoring $(n=1)$. Non-conventional management was not included here unless it was felt to have adversely affected the outcome.

\section{DISCUSSION}

The specific sub-algorithm for bradycardia is shown in fig 1 . It draws heavily upon the differential diagnosis and management strategies presented in the reports because they specifically deal with bradycardia within the context of anaesthesia.

Analysis of the AIMS reports shows that, in the majority of cases, bradycardia is managed appropriately. It is clear, however, that deficiencies in problem solving which have been identified in other arenas also exist in anaesthesia. For example, ARC guidelines on basic and advanced cardiac life support are clear on the institution of ECC in pulseless states. On a number of occasions in the reports there was a failure to interpret loss of peripheral pulses as a cardiac arrest. Not enough information was available to attempt to associate this with a poor outcome. However, it was interesting to observe

\begin{tabular}{|c|c|}
\hline Outcome & No $(\%)$ of cases \\
\hline $\begin{array}{l}\text { Good } \\
\text { Death on table } \\
\text { Death postoperatively } \\
\text { Intensive care admission* } \\
\text { High dependency admission } \\
\text { Coronary care admission } \\
\text { Cardiac eventt } \\
\text { Neurological event } \\
\text { Surgery abandoned } \\
\text { Otherf } \\
\text { Not specified } \\
\text { Total }\end{array}$ & $\begin{aligned} & 165(63) \\
& 8(3) \\
& 14(5) \\
& 19(7) \\
& 2(<1) \\
& 3(1) \\
& 12(5) \\
& 2(<1) \\
& 4(2) \\
& 5(2) \\
& 31(12) \\
& 265(100)\end{aligned}$ \\
\hline \multicolumn{2}{|c|}{$\begin{array}{l}\text { *Intensive care admission with recovery specified or outcome } \\
\text { not specified. } \\
\text { tIncludes ECG evidence of myocardial ischaemia or } \\
\text { pulmonary oedema but not admission to coronary care unit. } \\
\ddagger \text { Aspiration }(n=1) \text {, awareness }(n=1) \text {, chest drain }(n=1) \text {, } \\
\text { persistent postoperative hypoxaemia }(n=1) \text {, tracheostomy } \\
(n=1) \text {. }\end{array}$} \\
\hline
\end{tabular}

that, in approximately half of the cases of cardiac arrest in which ECC was not described, cardiovascular collapse followed an initial presentation of bradycardia with hypotension. The conventional management of asystole includes early adrenaline. Atropine is recommended as a second line agent in refractory asystole or in the initial management of bradycardia associated with hypotension. The AIMS reports describe several cases of cardiac arrest in which a successful outcome was achieved with the use of atropine instead of adrenaline. Atropine without ECC was used successfully in five cases of reflex induced sinus arrest. If necessary, cardiac pacing should be instituted. ${ }^{10}$

The sub-algorithm begins with a reconsideration of the core algorithm COVER ABCD-A SWIFT CHECK as an initial response, as the causes of $20 \%$ of cases of bradycardia can be expected to be diagnosed as a result. The high incidence of airway or ventilation related events lends further support to this approach. It is easy to visualise the difficulties and delays one might encounter in managing a crisis situation in which it is not clear whether the primary problem is airway, heart rate, or blood pressure. COVER ABCD-A SWIFT CHECK enables cross referencing with airway related problems which are represented on other pages of the crisis manual. This establishes a system by which airway problems can be diagnosed and managed definitively at the same time that supportive measures to restore heart rate and blood pressure are instituted. This is important because aggressive resuscitation of the cardiovascular system may follow an episode of

Table 7 Bradycardia: management

\begin{tabular}{|c|c|c|c|c|c|c|c|}
\hline \multirow[b]{2}{*}{ Grade } & \multirow[b]{2}{*}{ Description } & \multicolumn{5}{|c|}{ Presentation group (n) } & \multirow[b]{2}{*}{ Total } \\
\hline & & CA & CAP & $\mathrm{H}$ & $\mathbf{N}$ & HY & \\
\hline 1 & Conservative* & 3 & 0 & 17 & 19 & 0 & 39 \\
\hline 2 & Atropine \pm 1 & 15 & 5 & 46 & 34 & 0 & 100 \\
\hline 3 & Vasopressors \pm 2 & 2 & 1 & 33 & 0 & 0 & 36 \\
\hline 4 & Adrenaline $\pm \overline{3}$ & 17 & 6 & 11 & 0 & 0 & 34 \\
\hline 5 & Antidysrhythmic/pacing agents, cardiac pacing \pm 4 & 1 & 1 & 2 & 0 & 0 & 4 \\
\hline$E$ & External cardiac compression & 22 & 7 & 2 & 0 & 0 & 31 \\
\hline$M$ & Increase monitoring & 0 & 0 & 3 & 0 & 0 & 3 \\
\hline NC & Non-conventional & 0 & 5 & 5 & 1 & 0 & 11 \\
\hline NS & Not specified & & & & & & 40 \\
\hline
\end{tabular}




\section{Key messages}

- From the first 4000 reports to AIMS, 265 reports (7\%) which described bradycardia were studied; $84 \%$ of these were adults and 16\% were children under 14 years of age.

- More than half $(62 \%)$ were graded ASA I or II.

- Bradycardia occurred most commonly in the maintenance phase $(61 \%)$.

- Among 319 reported causes, the most common were drug events $(28 \%)$, airway or ventilation problems $(16 \%)$, autonomic reflexes $(14 \%)$, epidural or spinal anaesthesia (9\%).

- Airway events were the predominant cause of bradycardia in children (47\%). Hypotension was less common $(21 \%)$ but cardiac arrest more common $(32 \%)$ than in adults.

- Cardiopulmonary events causing bradycardia are more likely than other causes to be associated with cardiac arrest.

- Neuraxial blockade causing bradycardia had the commonest association with hypotension.

- Surgical events were associated with 13 reports (5\%): orthopaedic reaming, cementing, various manipulations, pneumoperitoneum.

- Bradycardia was "sinus" in 79\%, "nodal" in 11\%, and "complete heart block" in 5\%. Degeneration into asystole occurred in 19 cases (7\%).

- Death occurred on the operating table in eight cases and postoperatively in 14 cases. ICU admission occurred in 19 cases $(7 \%)$.

- In the 25 cases of "non-conventional" management, the inappropriate use of drugs or techniques during resuscitation (for example, calcium, isoprenaline, omission of indicated adrenalin, DC cardioversion for asystole) was most common.

- While the core algorithm managed 45 cases $(17 \%)$ adequately, the specific bradycardia sub-algorithm was successful for all but two of the remainder.

hypoxaemia and the possibility of multiple causes for bradycardia will always need to be considered.

In a similar manner to the airway, there is the potential for concentration on one component of the problem to the neglect of the wider problem when bradycardia occurs with either hypotension, hypertension, or cardiac arrest. Even when using an algorithm-based approach, the question may be raised "On which page do I start?" The AIMS reports were analysed in an attempt to identify presentation profiles from which high yield diagnostic and management pathways could lead. Several conclusions were drawn.

It is not possible to predict whether bradycardia is the primary or secondary event from the outset. This is a retrospective diagnosis made on the understanding of the pathophysiology after a diagnosis has been made. In some instances it is never possible to determine, for example, sepsis, bone cement and cardiopulmonary events.

The significance of any decrease in heart rate will be determined by the associated blood pressure rather than the absolute heart rate. When bradycardia occurs and the patient is pulseless, then go to the cardiac arrest algorithm because early aggressive management is required (table 4 ). If the rhythm is reflex induced sinus arrest, then the most effective treatment is to cease the precipitating event. If the patient is hypotensive it is worth noting the causes of hypotension and considering the use of vasopressors and aggressive fluid therapy. This is equally important in the context of spinal, epidural, ${ }^{11}$ or regional blockade (especially interscalene block $^{12}$ ). This can be achieved by reviewing the hypotension sub-algorithm. However, it should remain a problem of rate until the diagnosis has been made because of the important role of atropine and tertiary management options which include transvenous pacing. If the patient is hypertensive, then the problem should be managed according to the hypertension sub-algorithm.

Another issue which has the potential to complicate the problem is whether the prevailing cardiac rhythm should be diagnosed early. Sinus and non-sinus rhythms were compared with respect to ASA grading, haemodynamic status at the time of diagnosis, management. and outcome. This was done in an attempt to determine whether an early diagnosis of rhythm would be of value in the overall management of the problem. No differences were apparent. Analysis of cardiac rhythm should therefore not be pursued to the exclusion of supportive therapy or diagnosis of possible causes.

\section{ACKNOWLEDGEMENTS}

The authors would like to thank all the anaesthetists in Australia and New Zealand who contributed to the 4000 incident reports upon which this and the other 24 papers in the Crisis Management Series are based. The coordinators of the project also thank Liz Brown for preparing the draft of the original Crisis Management Manual; Loretta Smyth for typing; Monika Bullock RN for earlier coding and classifying of data; Dr Charles Bradfield for the electronic version of the algorithms; Dr Klee Benveniste for literature research; Drs Klee Benveniste, Michal Kluger, John Williamson and Andrew Paix for editing and checking manuscripts. Dr Craig Morgan carried out detailed review of cases and was an author of the initial draft of this paper but declined authorship of the final draft. He is thanked for having done the original hard work and participating in the development of the manuscript.

\section{Authors' affiliations}

L M Watterson, Senior Staff Specialist and Director, Sydney Medical Simulation Centre, Royal North Shore Hospital, St Leonards, New South Wales, Australia

R W Morris, Director, Research and Development, Sydney Medical Simulation Centre, Royal North Shore Hospital, St Leonards, New South Wales, Australia

R N Westhorpe, Deputy Director, Department of Paediatric Anaesthesia and Pain Management, Royal Children's Hospital, Parkville, Victoria, Australia

J A Williamson, Consultant Specialist, Australian Patient Safety Foundation and Visiting Research Fellow, University of Adelaide and Royal Adelaide Hospital, Adelaide, South Australia, Australia

This study was coordinated by the Australian Patient Safety Foundation, GPO Box 400, Adelaide, South Australia 5001, Australia.

\section{REFERENCES}

1 Runciman WB, Webb RK, Klepper ID, et al. Crisis management: validation of an algorithm by analysis of 2000 incident reports. Anaesth Intensive Care 1993;21:579-92.

2 Webb RK, Currie M, Morgan CA, et al. The Australian Incident Monitoring Study: an analysis of 2000 incident reports. Anaesth Intensive Care 1993;21:520-8.

3 Webb RK, van der Walt JH, Runciman WB, et al. Which monitor? An analysis of 2000 incident reports. Anaesth Intensive Care 1993;21:529-42.

4 Runciman WB, Kluger MT, Morris RW, et al. Crisis management during anaesthesia: the development of an anaesthetic crisis management manual. Qual Saf Health Care 2005; 14:e1.

5 Emergency Cardiac Care Committee and Subcommittees, American Heart Association. Guidelines for cardiopulmonary resuscitation and emergency cardiac care. Part III: Adult advanced cardiac life support. 1992 National Conference on Cardiopulmonary Resuscitation (CPR) and Emergency Cardiac Care (ECC). JAMA 1992;268:2199-241. 
6 Kloeck W, Cummins RO, Chamberlain D, et al. The Universal Advanced Life Support Algorithm. An Advisory Statement from the Advanced Life Support Working Group of the International Committee on Resuscitation. Circulation 1997;95:2180-2.

7 The Advanced Life Support Committee of the Australian Resuscitation Council. Adult advanced life support algorithm, July 2002. Available from the Australian Resuscitation Council at http://www.resus.org.au (accessed 12 September 2004).

$8 \mathrm{O}^{\prime}$ Connell T. Update in paediatric cardiopulmonary resuscitation. In: Keneally J, ed Australasian anaesthesia. Melbourne: ANZCA, 1994:27-37.

9 The Advanced Life Support Committee of the Australian Resuscitation Council. Paediatric advanced life support algorithm, March 2004. Available from the Australian Resuscitation Council at hitp://www.resus.org.au (accessed 12 September 2004).
10 Toprak V Yentur A, Sakarya M. Anaesthetic management of severe bradycardia during general anaesthesia using temporary cardiac pacing Br J Anaesth 2002;89:655-7.

11 Lesser JB, Sanborn KV, Valskys R, et al. Severe bradycardia during spinal and epidural anesthesia recorded by an anesthesia information management system. Anesthesiology 2003;99:859-66.

12 Sia S, Sarro F, Lepri A, et al. The effect of exogenous epinephrine on the incidence of hypotensive/bradycardic events during shoulder surgery in the sitting position during interscalene block. Anesth Analg 2003;97:583-8.

13 Australian Patient Safety Foundation. Crisis Management Manual: COVER $A B C D$ A SWIFT CHECK. Adelaide: Australian Patient Safety Foundation, 1996, 74 pp. Available at http://www.apsf.net.au/anaesthesia.htm (accessed 6 September 2004). 